\title{
Investigación y práctica reflexiva como categorías epistemológicas del desarrollo profesional docente.
}

\section{Research and reflective practice as epistemological categories of teacher development}

\section{Pesquisa e prática reflexiva como categorias epistemológicas de formação de professores}

Wilmer Hernando Silva Carreño*

Universidad de San Buenaventura, Bogotá, Colombia

Secretaría de Educación Distrital, Bogotá, Colombia

Resumen. Este artículo de reflexión procura entrever cómo, dado que la reflexión epistemológica es fundamental en todos los procesos del ámbito escolar, llevada a la pregunta por el conocimiento profesional docente, implica descentrar la discusión en torno a su formación y el desarrollo profesional como elementos fundante en la construcción de su conocimiento. En este proceso emerge la investigación como una

* Artículo de reflexión resultado del proyecto de investigación doctoral Formación y desarrollo de las capacidades. Perspectivas para una formación humanista desde el yo puedo fenomenológico y la teoría de Martha C. Nussbaum, desarrollado por el autor en el marco del programa Doctorado Interinstitucional en Educación, Universidad Pedagógica Nacional, 2013.

** Licenciado en Filosofía y Magíster en Ciencias de la Educación, Universidad de San Buenaventura, Bogotá; Doctorado Interinstitucional en Educación (en curso), Universidad Pedagógica Nacional. Docente Universidad de San Buenaventura, Bogotá; Docente Secretaría de Educación Distrital, Bogotá. E-mail: wsilva@usbbog.edu.co 
categoría que articula y pone de manifiesto que el profesorado en su formación y ejercicio deviene sujeto investigador, especialmente mediante la reflexión sobre sobre su propia práctica, que denota por tal un proceso de cuestionamiento continuo sobre su quehacer, no tanto sobre la práctica sino sobre el sentido de la misma. En lo que sigue se pretende hacer una aproximación al sentido de la investigación y la práctica reflexiva en el desarrollo profesional del profesor.

Palabras clave. Docente, investigación, práctica reflexiva, formación, desarrollo profesional (Tesauro Unesco).

\begin{abstract}
This article seeks to glimpse how reflection since epistemological reflection is central to all processes of school, led to the question of teacher professional knowledge, decentering involves the discussion of training and professional development as foundational elements in the construction of teachers' professional knowledge. In this research process emerges as a category that articulates and demonstrates that teachers in their training and exercise researcher becomes subject, especially by reflecting on their own practice, denoting by such a process of continuous questioning about his work, not so much on the practice but the meaning of it. In what follows is intended to approximate the direction of research and reflective practice in teacher professional development.
\end{abstract}

Keywords. Teaching, research, reflective practice, training, professional development (Thesaurus Unesco).

Resumo. Este artigo pretende vislumbrar como reflexão, uma vez que a reflexão epistemológica é essencial em todos os processos da escola, levou à questão do conhecimento profissional dos professores, descentramento envolve a discussão sobre a sua formação e desenvolvimento profissional, como elementos fundamentais na construção de seu conhecimento. Neste processo de pesquisa surge como uma categoria que articula e demonstra que os professores em formação e exercício torna-se sujeito de pesquisa, especialmente refletindo sobre sua própria prática, denotando por um tal processo de questionamento contínuo sobre o seu trabalho, não tanto sobre a prática, mas sobre o significado da mesma. No que se segue destina-se a aproximar a direção da pesquisa e prática reflexiva no desenvolvimento profissional dos professores. 
Palavras chave. Ensino, pesquisa, prática reflexiva, a formação, o desenvolvimento profissional (Tesauro Unesco).

\section{Aproximación epistemológica al desarrollo profesional docente}

La pregunta por aquello que sedimenta epistemológicamente la formación docente trae consigo la idea de que se ha definido al docente como un intelectual, como un trabajador de la cultura que piensa y produce conocimiento. Cómo es que el profesor es un intelectual, cómo es que es un sujeto que piensa y que produce conocimiento, son asuntos que reclaman un inquirir epistémico (Perafán y Aduriz, 2005, p. 65).

Se trata de reducir el riesgo al maestro a un técnico, un agenciador de un saber que se produce en los márgenes de la escuela. Frente a esto, emergen como categorías de fundamentación epistémica la comprensión del docente como profesional reflexivo y como profesional capaz de hacer de un conocimiento disciplinar, un conocimiento enseñable, posibilitar la trasposición de las disciplinas al aula, o lo que Garritz y Velasco (2004) referencian como trasposición didáctica, esto es, "el 'trabajo' que transforma un objeto de saber para enseñar en un objeto de enseñanza" (p. 4). Pero conviene diferenciar dos perspectivas de comprensión de lo disciplinar. Por una parte, se da una caracterización de la disciplina en el sentido expuesto por Foucault como aquello que mantiene un orden social; por otra, emerge el sentido de lo disciplinar como aquello susceptible de enseñabilidad, como saberes que están para ser enseñados y aprendidos.

Hay que indicar que el saber del profesor, el conocimiento del profesor, es totalmente distinto de la disciplina. Ésta puede ser un fundamento de contenido pero no el conocimiento del docente. De ahí que el conocimiento profesional del profesor tiene una fundamentación y comprensión epistemológica distintas de las disciplinas, aun cuando el quehacer docente deviene como trasposición de la disciplina hacia el conocimiento para ser enseñado, es decir, se configura el conocimiento disciplinar en la escuela como un conocimiento situado, contextualizado, de modo que la escuela no deviene como un lugar de transmisión del conocimiento disciplinar de manera objetiva, allí se transforma. Esto indica la importancia epistémica del conocimiento pedagógico del contenido como categoría de estudio de la formación docente (Salazar, 2005). De 
hecho, Ponte (2012) pone de manifiesto que el conocimiento profesional y el desarrollo profesional del profesorado son asuntos complementarios, atravesados por un proceso de formación disciplinar apropiado, competencia didáctica, reconocimiento del estudiante y del contexto, práctica profesional e investigación sobre la práctica, en efecto, "el contenido específico de la formación tiene una influencia fundamental en cómo ésta se diseña y desarrolla" (Badia y Monereo, 2004, p. 50).

Así, si la epistemología del conocimiento del profesor es distinta del conocimiento disciplinar, cabe preguntar cuáles son los procesos que explican la producción del conocimiento disciplinar y del conocimiento escolar o situado. Caracterizar el conocimiento personal que los profesores usan en su enseñanza, el conocimiento práctico personal, como lo desarrolla Pinchas (2005), puede ser un referente de comprensión al respecto, pero como tal, es asunto de una ulterior reflexión epistemológica.

Este análisis del fundamento epistemológico del conocimiento profesional docente trae consigo la idea de que el profesor es un investigador. El profesor tiene una condición histórica de producción de conocimiento, es decir, el concepto de la profesión docente no se reivindica sólo desde el mejoramiento del orden laboral, sino también, desde la posibilidad de pensar que produce conocimiento. Así, al indicar que es investigador denota que, más allá de su quehacer como enseñante, deviene investigador cuando reflexiona y piensa su labor docente. Con esto, se entrevé otro asunto importante relacionado con el hecho de que el profesor, más allá de comprenderse como un agenciador o transmisor de conocimiento, tiene un conocimiento que le es propio, pero esto debe clarificarse, es decir, ¿̇uál es el proceso por el cual se produce dicho conocimiento?

Un punto de partida para responder a esto puede fundarse en la comprensión misma del docente como investigador, como sujeto epistémico de su propio quehacer, formación y profesionalización. Así, emerge la cuestión de cómo se piensa críticamente la formación docente del profesorado, cómo se mira en el contexto actual la formación docente, en efecto, señala Freire: "Lo que se necesita es que el profesor, en su formación permanente, se perciba y se asuma, por ser profesor, como investigador" (Freire, 1997, p. 48).

En este sentido, conviene anotar que pensar un sustrato epistémico de la formación docente implica, entre otros aspectos, la necesidad de 
dar fuerza a la reflexión sobre la práctica docente para comprender la complejidad del proceso educativo, en efecto "el profesorado aprende a partir de su actividad y de la reflexión en torno a ella" (Ponte, 2012, p. 83), es decir, la práctica docente debe formar parte esencial de la formación inicial del profesorado, por lo que es necesaria la articulación del análisis de la propia practica con las aportaciones de la investigación y la innovación de la formación docente.

Esto no niega que la formación docente implique asegurar una formación adecuada en contenidos, pero pone de manifiesto que una formación que tome en cuenta una base epistemológica sólida, conlleva tanto pensar un giro del aprendizaje de contenidos al aprendizaje de procesos, como un enfoque de despliegue de las capacidades en la formación del profesorado. Se hace referencia a las capacidades en respuesta a la positivización de la formación docente que hace énfasis en la adquisición de competencias, lo que conlleva al problema de cómo conseguir que los profesores tengan la competencia profesional de orientar a los alumnos, o ¿̇cuáles son las competencias que permiten a los profesores desarrollar y evaluar competencias generales y especificas en sus alumnos? Si existe un diseño competencial para la enseñanza, ¿qué sujeto puede apropiarlo y por qué, entonces, no se habla de una educación exitosa? ¿Por qué la calidad educativa es, especialmente en el contexto colombiano, un asunto pensado a futuro?

Una posible respuesta a lo antes descrito puede partir de incorporar los resultados de la investigación en didáctica sobre el desarrollo profesional docente para diseño curricular de la escuela. Esto implicaría, por una parte, el desarrollo de competencias en análisis didáctico de los procesos de enseñanza, como una metacompetencia, sin descuidar el conocimiento para ser enseñado; y por otra, generar propuestas de formación permanente del profesorado que ayuden a los profesores en ejercicio a desarrollar esta metacompetencia.

Un camino sería lograr proyectos que permitan analizar el impacto de los modelos de formación en la transformación de las prácticas en el aula, desde luego, conviene analizar qué distancia hay entre los resultados de las investigaciones educativas y la práctica docente y los resultados de las trasformaciones de práctica en el aula. Siguiendo a Ponte (2012), hay que indicar que, precisamente: 
[...] la investigación, pensada como la forma por excelencia de construcción de conocimiento, cuando está orientada a los problemas de la práctica profesional puede ayudar a identificar estrategias de resolución de estos problemas y asumir un efecto formativo de gran alcance sobre los respectivos participantes (p. 94).

Pensar entonces la formación docente y el mismo desarrollo profesional docente retrotrae la importancia de ir más allá de la gestión de políticas de formación continuada o remedial que han desbocado en suplir espontáneamente las deficiencias docentes en su ejercicio, sin atender a un plan integral de desarrollo profesional docente. En efecto, se cae en la ambigüedad de valorar el desarrollo profesional docente a partir de los resultados en el aprendizaje de los estudiantes, esto es, si los estudiantes evidencian que aprenden, generalmente, se llega a afirmar que el profesor ha aprendido.

Esta dificultad en la que se pretende establecer el desarrollo profesional docente a partir de los resultados de los estudiantes o a partir de pruebas externas conlleva a cuestionar, por ejemplo, ¿̇cuáles son los referentes o criterios que indican que el profesorado está aprendiendo en la formación continuada? La formación docente, pues, se ha de resituar como un elemento especial y central de lo educativo, se ha de posibilitar la puesta en escena del valor de la formación, especialmente, inicial. Esto implica, sí, fortalecer una perspectiva cognitiva, disciplinar, en la formación docente, pero no puede darse al margen de una perspectiva comunitaria, social, esto es, integrar el ámbito contextual del ejercicio docente. Con esto es plausible indicar que una aproximación epistemológica del conocimiento y desarrollo profesional docente, como lo indica Perafán (2004), es idiosincrásica, esto es: "una epistemología local, enfocada principalmente en las reglas de funcionamiento propio y en las referencias específicas que intervienen en la constitución y en la producción del conocimiento de cada profesor" (p. 84).

\section{La pregunta por la investigación}

El desarrollo profesional del profesorado se ha configurado como un complejo campo de reflexión epistemológica, que intenta ir más allá de repensar la formación de competencias disciplinarias. Implica establecer una reinterpretación del sentido de los elementos, situaciones, y problemas que devienen en la formación. Pero este carácter epistemológico no 
es un intento de validar o interpretar el desarrollo e incidencia de teorías pedagógicas en el desarrollo profesional y las prácticas docentes. Pensar y posibilitar el desarrollo profesional del profesor no aguarda un proceso de perfeccionamiento disciplinar y operativo de las prácticas de un docente, no es un simple proceso de actualización cognitiva, pedagógica o procedimental, no es un espacio de formación bajo la intencionalidad de procurar sujetos competentes en saberes específicos, aunque sí los implica, por cuanto constituye, ante todo, un constructo problematizante de la cotidianidad pedagógica que atañe a la educación como estructura social y el papel del desarrollo profesional del profesor frente a ésta.

Es claro, entonces, que el desarrollo profesional del profesor no se refiere a unos procesos por el cual se puede acceder a un constructo de conocimientos, donde éste, el conocimiento, se ve como un bien deseable y conveniente, configurándose así un modo de enciclopedismo profesional y disciplinar. Es esta una conceptualización cerrada del conocimiento y del desarrollo profesional del profesor. Éste último debe interrogar lo que sucede en las bases estructurales del poder, de la sociedad y del existir humano del profesor, discerniendo las caracterizaciones socioculturales del contexto en que deviene las prácticas pedagógicas del docente, y los condicionamientos propios en cada ámbito contextual. Por esto, una formación epistemológica emerge como un elemento a partir del cual el docente se vea ya no como un mero agenciador de conocimiento, sino como un sujeto productor de conocimiento.

Aun cuando, incluso, en la investigación en educación se entrevé cierta tendencia a abordar los problemas susceptibles de la misma bajo un enfoque cuantitativo o cuando menos, bajo un enfoque cualitativo, pero, como señala Ponte (2012), "casi siempre [a partir de] estudios de casos que pronto relegan a un segundo plano las cuestiones filosóficas sobre la naturaleza del [saber]" (p. 84). Esto trae consigo la pregunta de cómo trascender los resultados de dichos estudios de casos más allá de sus fronteras, de modo que se conviertan en referentes, sino teóricos a lo menos pragmáticos, para una mejor comprensión del conocimiento y ejercicio docente.

El mismo Ponte (2012), reseñando un trabajo del año 1992, señala que: “... surge la necesidad de elaborar trabajos de naturaleza más teórica, que ayuden a precisar el significado de concepciones, creencias, conocimiento y saber y a sistematizar los resultados empíricos que se van 
obteniendo" (p. 84). Se reclama con esto la necesidad de evidenciar procesos de articulación entre el devenir de lo educativo, en este caso, el desarrollo profesional del profesor, con los resultados o la producción científica de los estudios científicos que se han hecho al respecto. A partir de esto puede entreverse la importancia de lo enunciado por Ponte, quizá a manera de tesis, sobre el sentido de la investigación en el desarrollo profesional del profesor, a saber:

La investigación, pensada como la forma por excelencia de construcción de conocimiento, cuando está orientada a los problemas de la práctica profesional puede ayudar a identificar estrategias de resolución de estos problemas y asumir un efecto formativo de gran alcance sobre los respectivos participantes (Ponte, 2012, p. 94).

Sin embargo, aunque como lo afirma el mismo Ponte (2012), referenciando a Schön (1983): "la investigación científica en educación no puede dar cuenta de todas las complejidades de la práctica profesional [...]" (p. 86). Como se indicó anteriormente, la investigación constituye, en todo caso, un aporte estructurante y fundante del desarrollo profesional del profesor por cuanto puede brindar "pistas y orientaciones curriculares, examinando la naturaleza del [saber], analizando los procesos de construcción del aprendizaje, y sugiriendo conceptos centrales para observar, construir y conducir situaciones" inherentes a la práctica y el desarrollo profesional del profesor (Ponte, 2012, p. 91).

\section{Investigación y desarrollo profesional del profesor}

La formación epistemológica del profesor constituye un referente fundamental para pensar una ruta de desarrollo profesional sólida que permita una trasposición de las disciplinas hacia la gestión de procesos de transformación e intervención social, en el marco del devenir técnico y científico de la época actual. Emerge la necesidad de posibilitar la formación de un profesional que, bajo un enfoque epistemológico específico, se descubre ante todo como persona en su ejercicio profesional reflexivo, crítico, y ante todo, investigador, cuya función no es la de transmitir conocimientos mediante recetarios procedimentales o cuyo rol no se define por un carácter enciclopedista, sino que deviene como sujeto investigador activo y garante de procesos de transformación social. De ahí que conviene tener en cuenta que "no sólo basta con que al docente se le brinden todas las oportunidades para una adecuada preparación [...] 
también es necesario brindarle los espacios y tiempos para que ponga en práctica lo aprendido" (Padilla, 2008, p. 93).

Se requiere, entonces, considerar los fundamentos metodológicos y epistemológicos del pensamiento y los procesos de lo educativo, como un constructo riguroso y sistemático desde donde la investigación, las disciplinas, las ciencias, los métodos, los paradigmas; tienen la tarea de corresponder con la apremiante realidad que les circunda, esto es, no pueden estar al margen de la continua construcción social. Emerge en este sentido la investigación bajo un carácter de función sustantiva y como tal, en el quehacer del profesor procura la calidad de los procesos educativos y la búsqueda del desarrollo profesional y la práctica del profesor con carácter científico y crítico, de modo que los procesos educativos institucionales sean una respuesta a las necesidades y a los proyectos de desarrollo cultural y científico más allá de la institución escolar. El profesor como profesional y su comprensión de las ciencias de la educación y en cuyo ámbito deviene, no puede desentenderse de su rol como investigador, pero tampoco puede perderse en la complejidad del conocimiento científico al margen de los fenómenos sociales que devienen en su entorno.

El desarrollo profesional del profesor ha de aportar elementos referenciales para que el profesor instaure las bases para el análisis de los conceptos y los procesos de construcción de conocimiento y su validación dentro de las ciencias sociales y humanas. Se trata de realizar una reflexión sistemática sobre los elementos epistemológicos tanto de los sujetos como de los colectivos; tanto del saber como del conocimiento; tanto de las sociedades como de las culturas; como puntos de partida para establecer la plataforma sobre la cual se instaura y se valida el conocimiento en los diversos aspectos de la educación (pedagogía, enseñanza, didáctica, currículo, entre otros); como una perspectiva de análisis y salida a lo indicado por Vargas (2010) respecto del desarrollo de la ciencia: "Al supuesto de que el resultado de la investigación científica se revierte en desarrollo tecnológico se opone el hecho de que las tecnologías avanzan sin esquemas explicativos y crean la situación de retraso de las ciencias respecto a su desarrollo" (p. 6).

No es posible, por tanto, desconocer que la época contemporánea a la que asistimos se ve seriamente afectada o condicionada por el desarrollo de la técnica, la ciencia, la informática, la tecnología, y que como conse- 
cuencia de dichos procesos se ha establecido una orientación pragmática de la ciencia y un horizonte de deshumanización y desestructuración del conocimiento y del saber. Pero esta misma época caracteriza y configura, desde los propios niveles de conocimiento que produce, divulga, innova, sustituye y fortalece nuevas formas de comprensión del mundo, de lo humano, de saber y de discurso. ¿Cuál es la pertinencia de la fundamentación epistemológica en este contexto? La respuesta puede darse tomando como punto de partida que si es dado caracterizar y nominar una era de la sociedad del conocimiento, no se hace para comprender las formas de saber que se configuran sino ante todo para posibilitar la emergencia del sentido mismo de dichos saberes que se esconde en los flujos de producción y de innovación.

El desarrollo profesional del profesorado reclama así una convergencia epistemológica que tome como base la pregunta por el sentido del conocimiento (por ejemplo articulando el conocimiento erudito y el conocimiento de contenido didáctico) en función de dar solución a los asuntos de lo humano desde la educación. Es un constructo epistemológico de interdependencia entre el devenir del hombre, la emergencia contemporánea de inquietantes fenómenos sociales y la estructura y organización del saber en su producción, intercambio y consumo. El alcance de la convergencia entre saber, conocimiento y disciplina denota ya un horizonte de análisis, debate y reflexión. No es una pretensión articular una respuesta a esta inquietud, por cuanto el darse como tal es en sí su propio sentido, pero sí hay que indicar con Perafán (2004) que una "integración consciente de los referentes epistemológicos del profesor en la categoría de análisis epistemología polifónica del profesor, constituye una herramienta de análisis para los propios profesores" (p. 184).

\section{La práctica reflexiva en la formación y desarrollo profesional docente}

Pensar el desarrollo profesional docente trae consigo preguntar, en qué medida y cómo el docente se constituye, a la luz de lo indicado por Perrenoud (2004), en un practicante reflexivo. Se ha abordado atrás el sentido de la investigación como categoría fundante en la formación y el desarrollo profesional docente, indicando precisamente que el profesor deviene investigador cuando reflexiona, cuando piensa su labor docente. Pero cuando solo deviene como enseñante hay un distanciamiento, por tanto, de ser investigador y de ser un profesional reflexivo. Emerge, 
entonces, la necesidad de dar fuerza a la reflexión sobre la práctica para comprender la complejidad tanto del proceso educativo como de la formación y del desarrollo profesional docente, se entrevé, así, una práctica reflexiva sobre el quehacer docente.

En este sentido, la reflexión, la practica reflexiva, se comprenden, a partir de lo señalado por Perrenoud en el libro Desarrollar la práctica reflexiva en el oficio de enseñar, como elementos claves en el oficio de enseñar. Cada uno articula de manera específica la importancia de hacer del quehacer pedagógico un medio para construir en el docente una actitud reflexiva, que vuelve sobre sí para constituirse como dador de sentido. La reflexión indica distanciarse de aquello que se hace, piensa o proyecta para hacer conciencia de la totalidad de la situación tenida como objeto. Además, si el carácter antropológico de la reflexión es conciencia o conocimiento de sí mismo, reflexionar denota comprender de forma particular y compleja una situación. Por esto, la reflexión es retrospectiva y prospectiva, integra lo acontecido como hecho y como pensamiento y así mismo pone en continuo movimiento, se encamina hacia el futuro (Perrenoud, 2004, p. 35).

Según Perrenoud (2004), la noción de práctica reflexiva hace referencia tanto a la reflexión sobre cada uno de los elementos que hacen parte de un proceso, de una situación, como también al hecho de hacer de la misma reflexión el propio objeto de comprensión, esto es, reflexionar sobre la acción. Así, la práctica reflexiva entraña dos momentos: como reflexión en la acción, que es inmediata, a breve plazo, consistentemente relacionada con la eficiencia técnica; y como reflexión sobre la acción donde el docente, alejado de lo inmediato, puede esclarecer, comprender e interpretar (p. 37).

La reflexión debe ser, además, un proceso metódico y constante de manera que sean los hechos los que estén determinados y definidos por una actitud reflexiva y no al contrario, es decir, abandonar aquella actitud reflexiva como consecuencia de lo sucedido. Por esto la práctica reflexiva se fortalece y favorece mediante el habitus, mediante su continuidad. La postura reflexiva significa formar el habitus de la reflexividad, fomentar esquemas reflexivos, estar siempre en disposición de reflexionar para valorar y mejorar, tener conciencia de la reflexión, de su importancia, de sus procesos y de su evolución. Exige autonomía y direccionamiento propio de la reflexividad pero también integración de lo otro, es decir, 
comprensión de la totalidad de cada experiencia, constituyendo así una epistemología reflexiva. En efecto, el pensamiento reflexivo implica la consideración activa, persistente y cuidadosa de cualquier creencia o práctica tomando en cuenta las razones que la sostienen y las consecuencias que puede tener a futuro.

Ahora, ¿por qué es importante la practica reflexiva en el desarrollo profesional docente? Puede indicarse ante esto que una práctica reflexiva trasciende la superficialidad de la formación y la enseñanza, prepara para comprender y enfrentar cada contexto, favorece la cooperación, innova los procesos pedagógicos, pero ante todo, provoca la actitud y la capacidad de volver sobre sí. Pero reflexionar no es solo evocar o hacer consciente algo, implica ante todo crítica, análisis, un proceso de aprehensión de lo dado. El reto consiste, no tanto en prepararse mediante la reflexión para lo posterior, sino en integrar hábilmente la labor y práctica pedagógica como instrumento que construye continuamente. De ahí el carácter pragmático de la reflexión, se concretiza solamente en la práctica, tiene que ser orientada a resolver cuestiones explícitamente sensibles. En eso radica su sentido.

\section{Conclusiones}

¿Cuál es el sentido que guarda el desarrollo profesional del profesorado desde el punto de vista epistemológico? Sin la intención de aproximar una comprensión plural de la educación, se entrevé que dicho horizonte epistemológico anuda, respecto de la educación, un encuentro inter y transdisciplinar de las ciencias sociales y humanas en aras a dar respuestas a los problemas inherentes a la educación misma. Se trata, pues, de posibilitar una aproximación al sustrato científico del saber propio del desarrollo profesional docente, de modo que se puedan hacer evidentes las propiedades epistemológicas que caracterizan la investigación como rigurosa y sistemática.

Este carácter epistemológico polifónico conlleva a que desde el desarrollo profesional, el profesor debe constituirse en sujeto de conocimiento y productor del mismo; las aulas en espacios epistémicos o lugares de investigación y la escuela en el contexto generador de respuesta a las necesidades de su entorno, tomando en cuenta que, como afirma Ponte (2012): "la realización de proyectos de investigación de los participantes sobre su práctica deviene un poderoso dispositivo de apoyo al desarrollo 
profesional [...]" (p. 94), pero sobre todo que se trata de ir más allá de la elaboración de proyectos de investigación y asumir la investigación como una categoría resignificadora del desarrollo profesional y de la realización de la práctica docente.

Así, emerge la necesidad de establecer una sólida fundamentación epistemológica del conocimiento del profesor más allá de la disciplina, pasando por la comprensión del profesor como un intelectual, gestor de cultura; por lo que la necesidad de fundar una formación para potenciar el desarrollo profesional docente implica también pensar la práctica reflexiva en torno a dicho proceso.

La reflexividad, entendida como la capacidad de comprensión y conciencia de la labor docente, implica que las prácticas pedagógicas deben ser continuamente constructivas. Por esto es esencial integrar a las mismas el contexto propio en el que se desarrollan, esto es, valorar cada parte del momento o proceso, tener en cuenta los proyectos del otro, la intencionalidad del otro, valorar las alternativas, considerar las consecuencias de cada acción, en efecto, como indica Perrenoud (2004), "formar a un practicante reflexivo es ante todo formar a un profesional capaz de dominar su propia evolución, construyendo competencias y saberes nuevos o más precisos a partir de lo que se ha adquirido y de la experiencia" (p. 10).

\section{Referencias}

Badia, A. y Monereo, C. (2004). La construcción de conocimiento profesional docente. Análisis de un curso de formación sobre la enseñanza estratégica. En: Anuario de Psicología, vol. 35, n9 1, marzo 2004, pp. 47-70, Universitat de Barcelona.

Freire, P. (1997). Política y educación. México: Siglo XXI.

Garritz, A. y Velasco, R., (2004). El conocimiento pedagógico del contenido. En: Serie Educación Química 15 (2), abril de 2004.

Padilla, E. (2008). La formación del docente universitario con miras al desarrollo humano. En: Revista Educación y Desarrollo Social. Volumen II - No. 1, Enero - Junio de 2008, Bogotá.

Perafán G. y Aduriz, A. (2005). Pensamiento y conocimiento de los profesores. Debates y perspectivas internacionales. Bogotá: Editorial Nomos.

ITINERARIO EDUCATIVO • ISSN OIZI-Z753 • AÑO XXVII, N. ${ }^{\circ} 62$ • JULIO - DICIEMBRE DE 2OI3 • P. 24I-254 
Perafán, G. A. (2004). La epistemología del profesor sobre su propio conocimiento profesional. Bogotá: Universidad Pedagógica Nacional.

Perrenoud, P. (2004). Desarrollar la práctica reflexiva en el oficio de enseñar. Barcelona: Graó.

Pinchas, T. (2005). Conocimiento profesional y personal de los Profesores y de los formadores de profesores. Trad. de P. S. de Vicente Rodríguez. Profesorado, Revista de currículum y formación del profesorado, 9, 2. Universidad Hebrea de Jerusalén. Publicado originalmente en Teacher and Teaching Education, 7 (3), 1991, 263-268.

Ponte, J. (2012). Estudiando el conocimiento y el desarrollo profesional del profesorado de matemáticas. En: N. Planas (Coord.): Teoría, Crítica y Práctica de la Educación Matemática. Barcelona: Graó. pp. 83-98.

Salazar, S. (2005). El conocimiento pedagógico del contenido como categoría de estudio de la formación docente. En: Revista Electrónica Actualidades Investigativas en Educación. Volumen 5, Número 2, 2005.

Vargas, Germán (2010). Tratado de epistemología. 2a edición. Bogotá: San Pablo. 